\title{
The Radar Signature of the Wind Lens: a less disruptive wind turbine?
}

\author{
Alessio Balleri ${ }^{1}$, Allann Al-Armaghany ${ }^{2}$, Hugh Griffiths $^{2}$, Kenneth Tong ${ }^{2}$ and Takashi Matsuura ${ }^{3}$ \\ ${ }^{1}$ Informatics and Systems Engineering, Cranfield University, Defence Academy of the United Kingdom * \\ ${ }^{2}$ Electronic \& Electrical Engineering, University College London \\ ${ }^{3}$ Engrid Ltd, London
}

Keywords: radar clutter, radar interference, wind farm clutter, wind turbines.

\begin{abstract}
We study the radar signature of a new type of wind turbine, named the Wind Lens. This design includes a flanged shroud around the turbine which concentrates the wind flow past the turbine blades and hence improves the efficiency. The design also offers improved safety, and reduces acoustic noise. Furthermore, it may offer a significantly lower radar signature, which may make the design much more attractive for use in situations where conventional wind turbine designs may disturb the operation of radars. We present the results of an experimental trial, carried out in the UK, to measure the Radar Cross Section (RCS) of a $5 \mathrm{~kW}$ Wind Lens turbine prototype and we provide a reference database that can be used for comparing the Wind Lens RCS with that of conventional turbines. We investigate methods to further reduce the Wind Lens RCS and present the results of a time-varying Doppler analysis. Results show that the addition of a metallic mesh around the shroud obscures the rotating blades, and hence mitigates the RCS by $15 \mathrm{dBm}^{2}$, at angles where the radar interference is highest.
\end{abstract}

\section{Introduction}

Many countries are investing large sums of money to support the development of alternative and sustainable ecologically-friendly technologies, including wind farms, as a means to reduce reliance on fossil fuels and generation of greenhouse gases. The European Union has targeted that $20 \%$ of energy should be generated by renewable sources by 2020 , and wind power will be the dominant technology to achieve this target because wind farms are the most mature technology currently available. It is known that wind turbines can significantly disrupt the operation of surveillance radars used for air traffic control and for air defence as well as marine and weather radars [14]. Wind turbines present a large radar signature due both to the turbine tower, and to the rotating blades which induce a Doppler spread that depends on the blade tip speed, frequency and aspect

*This work was carried out when Alessio Balleri was with the Dept of Electronic \& Electrical Engineering at University College London. angle. Wind farms cause detection problems to radar systems both because of the increased clutter level and because of the obscuration of true targets behind the wind farm as a consequence of a raised detection threshold. This can have an impact on the safety of the services provided to aircraft and on the reliability of air defence. Currently, half of all wind farm developments in the UK face objections from aviation stakeholders and the Ministry of Defence (MoD) on the grounds of radar interference, obstruction or impact to low flying operations, and the situation is similar in many other countries.

In recent years there have been increasing research efforts to study the impact of windfarm clutter on radar systems [6] [5] [4] [12] [13] [9] [8]. These include solutions that require a more sophisticated signal processing at the radar receiver as well as the adoption of Radar Absorbent Material (RAM) to shield the turbine and reduce the reflected power. Despite all these efforts, at present, radar wind farm clutter remains an open problem.

Recent work at Kyushu University in Japan has led to the development of a new type of wind turbine design which has been named the Wind Lens [10] [11] [15]. The key feature of this design is the addition of a flanged shroud around the turbine which concentrates the wind flow past the turbine blades and hence improves the efficiency of the turbine. In addition, the wind-lens structure helps reduce acoustic noise usually created by bare tips of wind turbine rotors and provides improved safety by acting as a protective layer around the rotor [1]. A $5 \mathrm{~kW}$ prototype design has been built and evaluated. The blade diameter is 2.5 $\mathrm{m}$, the shroud diameter is $3.4 \mathrm{~m}$ and the power output at a wind speed of $11 \mathrm{~m} / \mathrm{s}$ is $3 \mathrm{~kW}$. This design has demonstrated substantial improvements in efficiency, by a factor 4-5, over conventional turbine designs with the same diameter. It may be expected that the addition of the shroud may also serve to reduce the radar signature of the Wind Lens, particularly if the turbine blades can further be shielded by a wire grid mesh. In this paper we present a summary of the results of an experimental trial undertaken in the UK to measure the Radar Cross Section (RCS) of a $5 \mathrm{~kW}$ Wind Lens turbine prototype. We investigate methods to reduce the Wind Lens RCS by studying variations of the turbine RCS, as a function of aspect angle and time, due to the addition of a metallic mesh a) around the flanged shroud and b) with a mesh extension covering the front of the turbine. We present the results of a set of simulations and those of 


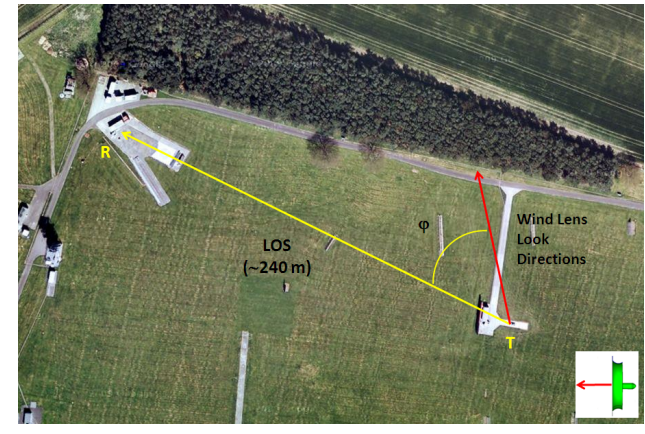

Figure 1: Experimental setup. The turbine (T) was placed about $240 \mathrm{~m}$ from the radar (R). The looking direction of the turbine with respect to the radar Line Of Sight (LOS), $\varphi$, was fixed at each measurements. The Wind Lens looking direction is shown by the unit vector indicating the orientation of the back of the turbine (as shown in the right bottom corner image).

a time-varying Doppler analysis of the Wind Lens turbine radar signature.

\section{Experimental Setup and Data Collection}

The experiments were carried out at the QinetiQ measurement range at Funtington, UK, in May 2011. The wind turbine was placed on a $10 \mathrm{~m}$ long mast about $240 \mathrm{~m}$ from the radar as shown in Fig. 1. The aspect angle $\varphi$ between the radar Line of Sight (LoS) and the unit vector indicating the orientation of the front of the turbine was controlled and remained fixed during each measurement. A network analyser was used to measure radar returns from the wind turbine with a sampling rate $f s=5 \mathrm{kHz}$ at $9.41 \mathrm{GHz}$ and 3 $\mathrm{GHz}$. In weak wind, the wind turbine rotor angular velocity was controlled by an external inverter and kept constant at 300 RPM (Revolutions Per Minute). In strong wind, the inverter failed to control the wind turbine and the rotor velocity varied around 300 RPM. Data was collected at $\mathrm{HH}$ and VV polarisations for three different scenarios: a) the original wind turbine; b) a stainless steel fine mesh grid (mesh size $1.35 \mathrm{~mm} \times 1.35 \mathrm{~mm}$ ) with $0.228 \mathrm{~mm}$ wire diameter fitted around the flanged shroud; c) the metallic mesh grid was further extended to cover the front of the turbine as shown in Fig. 2. Measurements were repeated for various aspect angles between 0 and 180 degrees. The signature of a $0.7 \mathrm{~m}$ side length trihedral characterised by an absolute RCS of $30 \mathrm{dBm}^{2}$ at $9.41 \mathrm{GHz}$, and $20 \mathrm{dBm}^{2}$ at $3 \mathrm{GHz}$, was measured for both the HH (Horizontal-Horizontal) and VV (Vertical-Vertical) polarization and used as a reference target to calibrate the wind turbine data.

\section{Results}

\subsection{Radar Cross Section Analysis}

The return from the Wind Lens turbine $s(t)$ was sampled at a rate $T_{s}=1 / f_{s}$ to generate the sequence $s_{r}(n)=s\left(n T_{s}\right)$ which was then recorded and processed offline with Matlab. The mean Radar Cross Section (RCS) of the turbine

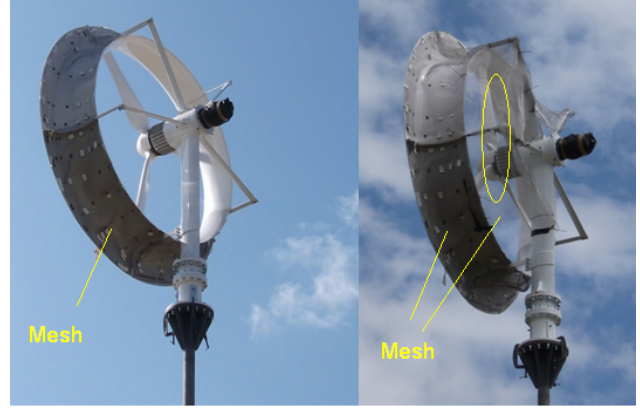

a)

b)

Figure 2: Photo of the Wind Lens turbine used for the measurements; a) with metallic mesh fitted on the flanged shroud and b) with metallic mesh on the shroud and an additional mesh extension to cover the front of the turbine.

for each aspect angle was calculated as

$$
\left.R C S=10 \log _{10}\left(\frac{1}{N} \sum_{i=1}^{N}\left|s_{r}(n)\right|^{2}\right)\right)-C,
$$

where $N$ is total number of sample of $s_{r}(n)$ and $C$ is the measured return from the calibrating trihedral. Table 1 shows a sample of calibrated turbine RCS values in both $\mathrm{VV}$ and $\mathrm{HH}$ polarizations at $9.41 \mathrm{GHz}$ for the original Wind Lens turbine, the Wind Lens turbine with fitted mesh and the Wind Lens turbine with extended mesh. Results show a variation of the RCS as a function of the aspect angle for all three scenarios. The RCS of the original Wind Lens turbine varies between $15 \mathrm{dBm}^{2}$ and $21 \mathrm{dBm}^{2}$ and that of the turbine with fitted mesh varies between $12 \mathrm{dBm}^{2}$ and 25 $\mathrm{dBm}^{2}$. The highest variation in RCS occurs in $\mathrm{HH}$ polarisation for the case with fitted mesh. The maximum value of the RCS corresponds to $\varphi=0$ degree aspect angle for the case with fitted mesh around the shroud. At 90 degrees aspect angle the addition of the mesh results in a RCS enhancement in both polarisations indicating an increase in the zero-Doppler component of the return. Values of the RCS on the average are stronger, for all aspect angles, in VV polarisation and for the case with fitted mesh. At both 105 at 135 degrees aspect angles the mesh extension results in a reduction of the RCS of up to about $8 \mathrm{dBm}^{2}$ in $\mathrm{VV}$ polarisation and $3 \mathrm{dBm}^{2}$ in $\mathrm{HH}$ polarisation. When the zero-Doppler component is removed from the turbine return ( by filtering the signal $s_{r}(n)$ with an ideal low-pass filter characterised by a cut-off frequency of $10 \mathrm{~Hz}$ ) to eliminate the contribution of the static structure, there is a considerable RCS drop in both $\mathrm{HH}$ and VV polarisations for all aspect angles and for all three scenarios. In this case we found that the RCS of the original Wind Lens turbine varies between $-4 \mathrm{dBm}^{2}$ and $2 \mathrm{dBm}^{2}$ with the lowest values occurring at 75 degrees and 90 degrees aspect angles for both polarisations. After zero-Doppler removal, the impact of the metallic mesh is evident. At 90 degrees the mesh obscures the rotating blades and the RCS drops below $16 \mathrm{dBm}^{2}$ for both polarisations. The RCS remains high at 0 degrees and 180 degrees, i.e. when the radar was facing the front of the blades. The drop in RCS due to the 


\begin{tabular}{|c|c|c|c|c|c|c|c|}
\hline & Angle [deg] & HH & VV & HH with mesh & VV with Mesh & HH with cover & VV with cover \\
\hline \multirow{3}{*}{ With 0 Doppler } & 90 & 12.4 & 13 & 14.9 & 17 & NA & NA \\
& 105 & 19.1 & 21.4 & 18.3 & 17.3 & 16 & 12.4 \\
& 135 & 15.6 & 15.6 & 19.4 & 19.9 & 14.1 & 13.9 \\
\hline \hline \multirow{3}{*}{ Without 0 Doppler } & 90 & -1.1 & -4.1 & -16.6 & -19.4 & NA & NA \\
& 105 & -1.2 & 0.4 & -2.4 & -0.5 & -7.9 & -13.3 \\
& 135 & -1.3 & -0.6 & 0.3 & -0.5 & -7.1 & -5.6 \\
\hline
\end{tabular}

Table 1: Sample of measured RCS.

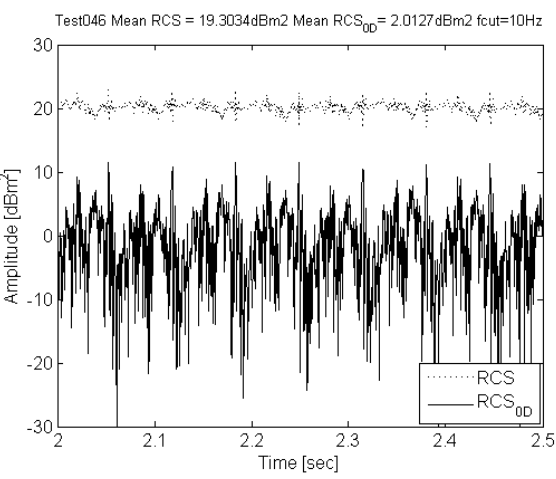

Figure 3: Wind turbine RCS as a function of time for data with zero-Doppler component (RCS) and without zeroDoppler component (RCSOD). These results correspond to an aspect angle of 45 degrees and $\mathrm{HH}$-polarised data.

metallic mesh around the shroud at 75, 105 and 135 degrees, i.e. close to 90 degrees, is almost negligible indicating that the mesh is not capable of obscuring the blades at such angles. This hypothesis is corroborated by the drop in RCS at 105 and 135 degrees when the mesh extension is added. Fig. 3 shows the wind lens RCS as a function of time for a 45 degree aspect angle when the blades were rotating at 300 RPM. The plot clearly shows that the RCS undertake a substantial variation depending on the blades position. They also show the drop in RCS between the case with zero-Doppler component and the case without zeroDoppler component. Results at $3 \mathrm{GHz}$ show the same trend as a function of aspect angle. Unfortunately, for a problem at the radar that occurred during the trials, the data were not calibrated with an acceptable level of confidence at this frequency and therefore it remained impossible to carry out an absolute comparison between polarisations and different scenarios. These results show that although the fitted mesh around the shroud increases the zero-Doppler component of the turbine signatures, it can be effectively deployed to obscure the rotating blades and hence mitigate the RCS at angles close to 90 degrees, i.e. where the radar interference due to the turbine blades is unacceptable. They also show that the Wind Lens presents RCS values that, after zero Doppler removal, are much lower than those of conventional turbines measured in previous experimental trials. These conventional turbines were much bigger (with 28-40 $\mathrm{m}$ long blades) and capable of power outputs in the order of the MegaWatts [14][6][12]. Although a fair comparison should be done given the same power output, these results indicate that the Wind Lens turbine is a favourable option in situations where lower power outputs are acceptable and conventional high-output turbines cannot be installed because they are either too big or would cause high radar interference. In the light of these results, work is in progress at Kyushu University to assess to what extent the addition of the metallic mesh on the shroud alters the efficiency of the turbine. Latest results have shown that the loss in performance due to the addition of a metallic mesh, with a mesh size of $3 \mathrm{~cm} \times 3 \mathrm{~cm}$ and a $2 \mathrm{~mm}$ wire diameter, is negligible when the mesh is placed at either the front or the back of the Wind Lens turbine. A much finer mesh, such that used in this experimental trial, may increase the wind resistance and lower the efficiency [2]. This will be assessed in detail at Kyushu University in future work.

\subsection{Time-Frequency Analysis}

The rotating blades and nacelle contribute to the generation of a complex return which is characterised by a timevarying Doppler spectrum. To study the properties of the Wind Lens turbine Doppler spectrum, the spectrogram $S(i, K)$ of $s_{r}(n)$ was calculated as the squared amplitude of the Short Time Fourier Transform (STFT) of $s_{r}(n)$ as

$$
\left|\sum_{n=1}^{N_{w}}\left\{s_{r}\left((i+1) \frac{N_{w}}{2}+n\right) w(n)\right\} e^{j 2 \pi \frac{n K}{N_{w}}}\right|^{2},
$$

where $N_{w}=2^{M}$ is the number of points of a suitable smoothing sliding window $w(n)$, and $K$ represents the digital frequency bin [7]. The time-varying Doppler analysis (i.e. the analysis of the frequency shifts due to the moving components of the turbine) and the study of the spectrogram is a tool that provides information on the Doppler spectrum properties of the interference from the turbine, such as bandwidth and Doppler shift, and how this varies as a function of time with respect to the position of the turbine components, such as the blades. It helps identifying the components that are responsible for producing the interference and it can provide useful insights to take actions to suppress their signature. Fig. 4 shows the spectrogram related to an aspect angle of 90 degrees for the case without and with metallic mesh fitted on the shroud. This was calculated by using a Hanning sliding window made of 64 samples, corresponding to a dwell time equal to $12.8 \mathrm{~ms}$. The plots show a significant variation of the Doppler components over time and a set of periodical flashes, due to the rotating blades, that cover the whole available Doppler spectrum at both 


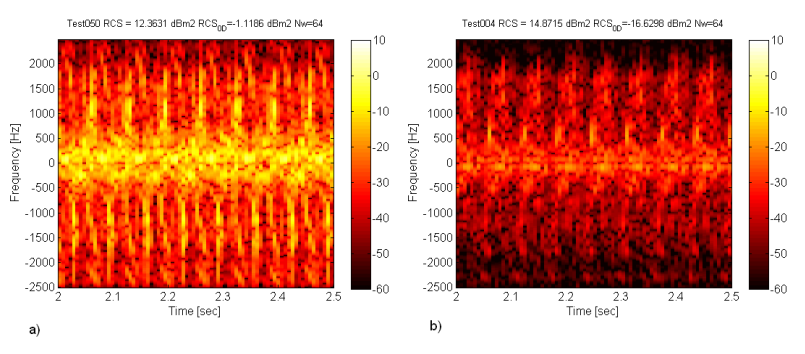

Figure 4: Spectrogram of the HH data for a 90 degrees aspect angle for a) turbine without fitted metallic mesh and b) turbine with fitted metallic mesh.

negative and positive Doppler shifts. These flashes correspond to the rotating blades being perpendicular to the radar line of sight (LOS), i.e. to instances of the target presenting a maximum radial velocity with respect to the radar LOS. The impact of the mesh on the wind turbine signature is evident. A comparison between the two plots show that the radar return from the blades can be significantly reduced by fitting the metallic mesh around the shroud up to values between at least $10-15 \mathrm{dBm}^{2}$. This is in agreement with the results of the mean radar cross section analysis presented in the previous section. The data at $3 \mathrm{GHz}$ presented the same behaviour.

\subsection{Simulation of the RCS of the turbine blade}

To further explore the scattering properties of the blade, its RCS was simulated with CST as a function of aspect angle [3]. The scenario parameters of the simulation were selected so that the 0 degrees aspect angle corresponded to the RCS of the blade in a position equivalent to the wind turbine being irradiated from the back ( $\varphi=0$ degrees). We then rotated the blade on its longer axis to obtain simulated RCS values over 360 degrees as shown in Fig. 5. The results, presented in Fig. 6, show that as expected the design of the blade results in a variation of the blade RCS by over $30 \mathrm{dBm}^{2}$ for both $\mathrm{X}$ band and $\mathrm{S}$ band and this is self-explicative of why the scattering levels in the spectrogram of the wind turbine returns vary as a function of the blade position. In particular, when the simulations are superimposed on the real data collected with an aspect angle $\varphi=45$ degrees, it can be shown that the maximum and negative maximum Doppler shifts occur for blade positions that correspond, in the simulation, to the blade being irradiated from 45 degrees (positive Doppler) and 135 degrees (negative Doppler). Results of the simulation show that the RCS of the blade is higher at 135 degrees and this corroborates the results of the time-varying analysis. These results suggest that, because the blades are the wind turbine components that are responsible of creating radar interference over a large bandwidth, its design needs to be thought so to maximise the wind turbine efficiency whilst reducing their radar RCS. The use of the metallic mesh or of a suitable material for the flanged shroud may results in an additional degree freedom under which the second requirement may be significantly relaxed.

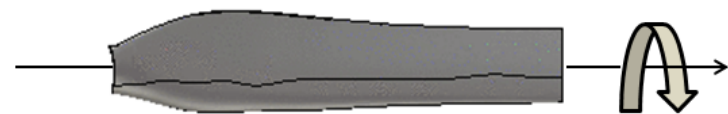

Figure 5: Blade position for a 0 degrees aspect angle. The blade is then rotated along its longer axes (indicated by the thin black arrow) and the RCS is calculated with CST with a step of 1 degree over 360 degrees. The image shows the CAD model of the blade that was imported into CST to carry out the simulations.
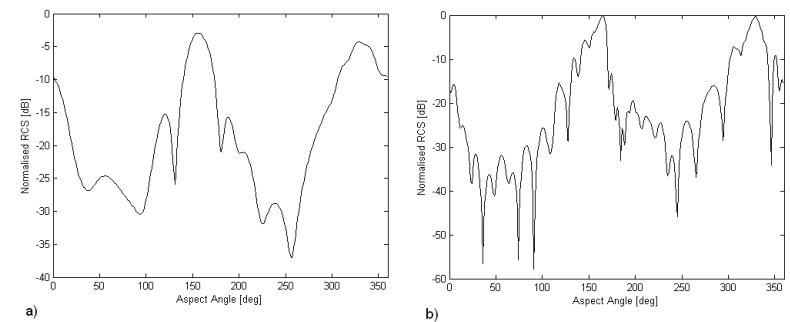

Figure 6: Simulated RCS of the Wind Lens blade at a) S band and b) X band. Data have been normalised with respect to the highest RCS (which is associated with the $\mathrm{X}$ band data).

\section{Conclusion and future work}

In this work the radar cross section of a $5 \mathrm{~kW}$ Wind Lens turbine prototype has been measured to allow comparisons with other turbine designs. Results of the Radar Cross Section analysis have shown that after zero-Doppler removal the Wind Lens turbine present a low RCS and that when a metallic mesh is fitted on the flanged shroud the RCS due to the rotating blades can be significantly reduced at angles close to 90 degrees. The time-varying Doppler analysis has corroborated that as expected the radar signature of the turbine is characterised by a time-varying Doppler spectrum. This is made of a set of flashes that cover a vast part of the Doppler bandwidth which occur when the blades are in a position perpendicular to the radar line of sight. The time-varying Doppler analysis has corroborated that the signature of the blades can be significantly reduced at aspect angles close to 90 degrees by fitting a metallic mesh around the shroud. It has also shown interesting reflecting properties associated with the blades that provide useful information that can be used to further reduced the RCS of the turbine. Results indicate that the Wind Lens turbine is a favourable option in situations where conventional high-output turbines cannot be installed, either due to space constraints or radar interference, and where lower power outputs are still acceptable. In addition to this, the results strongly suggest it will be possible to deploy similar solutions to a fitted metallic mesh to reduce the RCS of future Wind Lens turbine prototypes that will be capable of generating significantly higher power outputs. Considering that, given the same power output, the Wind Lens 
turbine design is 4-5 times smaller than conventional turbines it is likely that, with the deployment of a metallic mesh around the shroud or with the use of suitable material for the shroud, new Wind Lens turbines will present much lower Radar Cross Sections. A new 100 kW Wind Lens turbine prototype is under development and its RCS will be measured in future work.

\section{Acknowledgements}

The authors want to thank the Japanese Ministry of Environment (MoE) and Kyushu University (Prof Yuji Ohya, Dr Takashi Karasudani and Mr Kimihiko Watanabe) for funding this work. They also want to thank QinetiQ (Fungtinton, UK) for the support provided during the experimental trials.

\section{References}

[1] K. Abe, H. Kihara, A. Sakurai, E. Wada, K. Sato, M. Nishida, and Y. Ohya. An experimental study of tip-vortex structures behind a small wind turbine with a flanged diffuser. Wind and Structures, 9:413-417, September 2006.

[2] A. Balleri and Y. Ohya. The impact of a metallic mesh on the wind lens performance. private communication, 2011.

[3] CST. 2012 user manual. 2012.

[4] B. Gallardo-Hernando, J.M. Muñoz Ferreras, and F. Pérez-Martínez. Super-resolution techniques for wind turbine clutter spectrum enhancement in meteorological radars. IET Radar, Sonar and Navigation, 5(9):924-933, December 2011.

[5] B. Gallardo-Hernando, J.M. Muñoz Ferreras, F. Pérez-Martínez, and F. Aguado-Encabo. Wind turbine clutter observations and theoretical validation for meteorological radar applications. IET Radar, Sonar and Navigation, 5(2):111 -117, February 2011.

[6] B. Gallardo-Hernando, F. Pérez-Martínez, and F. Aguado-Encabo. Detection and mitigation of wind turbine clutter in c-band meteorological radar. IET Radar, Sonar and Navigation, 4(4):520 -527, August 2010.

[7] M. Greco, F. Bordoni, and F. Gini. X-band sea-clutter nonstationarity: influence of long waves. IEEE Journal of Oceanic Engineering, 29(2):269 - 283, April 2004.

[8] Yuchoi F. Lok, Alan Palevsky, and Jian Wang. Simulation of radar signal on wind turbine. IEEE Aerospace and Electronic Systems Magazine, 26(8):39-42, August 2011.

[9] A. Naqvi, Shang-Te Yang, and Hao Ling. Investigation of doppler features from wind turbine scattering. IEEE Antennas and Wireless Propagation Letters, 9:485 -488, May 2010.
[10] Y. Ohya and T. Karasudani. Development of a shrouded wind turbine with a flanged diffuser. Journal of Wind Engineering and Industrial Aerodynamics, 96(6):524-539, March 2008.

[11] Y. Ohya and T. Karasudani. A shrouded wind turbine generating high output power with wind-lens technology. Energies, 3:634-649, March 2010.

[12] J. Perry and A. Biss. Wind farm clutter mitigation in air surveillance radar. IEEE Aerospace and Electronic Systems Magazine, 22(7):35-40, July 2007.

[13] J. Pinto, J.C.G. Matthews, and G.C. Sarno. Stealth technology for wind turbines. IET Radar, Sonar and Navigation, 4(1):126 -133, February 2010.

[14] G.J. Poupart. Wind farms impact on radar aviation interests - final report. Technical Report FES W/14/00614/00/REP, QinetiQ, September 2003.

[15] T. Uchida and Y. Ohya. Micro-siting technique for wind turbine generators by using large-eddy simulation. Journal of Wind Engineering and Industrial Aerodynamics, 96:2121-2138, April 2008. 